\title{
Optimized Administration of Voriconazole and Therapeutic Drug Monitoring in Children and Adolescents: A Single-Centre Retrospective Experience from China
}

\section{Yi-Chang Zhao}

Second Xiangya Hospital of Central South University

\section{Yang Zou}

China Pharmaceutical University

\section{Dan Tang}

China Pharmaceutical University

\section{Chen-Lin Xiao}

Second Xiangya Hospital of Central South University

\section{Yi-Wen Xiao}

Second Xiangya Hospital of Central South University

\section{Feng Wang}

Second Xiangya Hospital of Central South University

\section{Bi-Kui Zhang}

Second Xiangya Hospital of Central South University

\section{Da-Xiong Xiang}

Second Xiangya Hospital of Central South University

\section{Feng Yu}

China Pharmaceutical University

Miao Yan ( $\nabla$ yanmiao@csu.edu.cn )

Second Xiangya Hospital of Central South University

\section{Research Article}

Keywords: Voriconazole (VRC), therapeutic drug monitoring, children, adolescents, China

Posted Date: February 26th, 2021

DOl: https://doi.org/10.21203/rs.3.rs-222933/v1

License: (c) (i) This work is licensed under a Creative Commons Attribution 4.0 International License. Read Full License 
Page $2 / 20$ 


\section{Abstract \\ Background}

Voriconazole (VRC) is a triazole anti-fungal agent and a first-line treatment for invasive fungal infection (IFI) generally. The purpose of our study was performed to explore the factors affecting voriconazole trough concentration $\left(\mathrm{C}_{\text {trough }}\right)$ and to show VRC dose adjustment experience in children.

\section{Methods}

The demographic information, concentration data, CYP2C19 genotypes and clinical outcomes of eligible children from January 1th, 2016 to December 31th, 2018 were collected. Factors affecting the voriconazole trough concentration were statistically analyzed.

\section{Results}

A total of 145 trough concentrations in 94 patients were included in this study, 54.5\% of which achieved the target concentrations; however, $35.9 \%$ and $9.6 \%$ of which were sub-therapeutic and super-therapeutic post-multiple dosing. For children $\leq 2,2-6,6-12$, and 12-18 years, the median VRC maintenance doses of $5.7,6.7,5.0$ and $3.3 \mathrm{mg} / \mathrm{kg}$ twice daily respectively had been required in order to achieve therapeutic level $(P<0.001)$. Co-administration of proton pump inhibitors affected VRC target trough concentration significantly $(P=0.001)$.

\section{Conclusion}

Younger pediatric patients might need a higher dosage regime to achieve therapeutic trough concentration. In order to ensure the effectiveness and safety of voriconazole in children, early and repeat monitoring of voriconazole is a powerful tool.

\section{Introduction}

2017 Updated guideline has demonstrated that children are at a high risk of invasive fungal diseases (IFD) undergoing allogeneic hematopoietic stem cell transplants (HSCT), leukemia, prolonged neutropenia and receiving high-dose corticosteroids ${ }^{1}$, which had made an important contribution to the proportion of morbidity and mortality ${ }^{2}$. Voriconazole is a second-generation triazole antifungal agent with broad antifungal spectrum including Candida, Aspergillus, Fusarium, Cryptococcus neoformans and Scedosporium $^{3,4}$. It was recommended by Aspergillus guidelines for first-line treatment of invasive aspergillosis and being widespread used in clinic $^{5}$. However, voriconazole dosing regimen has been particularly challenging for children. 
There are significant individual variations in the pharmacokinetics of voriconazole in children and adults. Several studies showed a significant concentration-dependent response between high steady-state plasma concentration and adverse events, between inadequate concentration and treatment failure $\mathrm{e}^{3,6,7}$. The steady-state voriconazole trough concentration was affected by various factors, such as age, ethnic, dosage, drug combination, genetic polymorphism of CYP2C19 enzyme and enzymes' activity. Hence, in order to ensure the efficacy of antifungal and reduce adverse reactions of voriconazole, routine and repeat therapeutic drug monitoring (TDM) of the steady-state voriconazole trough concentration was strongly recommended by the European Conference on Infections in Leukemia (ECIL) guidelines ${ }^{8}$. To date, no recommended voriconazole dosing is applicable to Asian children. With published narrow therapeutic range $(1.0-5.5 \mathrm{mg} / \mathrm{L})^{9}$, to achieve VRC exposures, 7 to $8 \mathrm{mg} / \mathrm{kg}$ intravenous twice daily of dose ranges were recommended in Caucasian children ${ }^{7}$. However, owing to lack of continually VRC TDM, a maintenance dose lower than the package insert manner was commonly prescribed in children patients in our hospital.

TDM of voriconazole in Asian children are not always available for clinician due to very limited studies. For now, no definite guidelines are available for voriconazole dose adjustment in pediatric patients. Therefore, we described our experience with voriconazole administration, dose adjustment and therapeutic drug monitoring. In this research, we retrospectively collected clinical outcomes of pediatric patients included through the electronic medical system at our hospital. The present study aimed (i) to investigate the relationship between steady-state voriconazole trough concentration and clinical response and adverse reactions, (ii) to explore the affecting factors on voriconazole trough concentrations and (iii) to study the impact of CYP2C19 genetic polymorphism on voriconazole metabolism in the growing child.

\section{Materials And Methods}

\section{Setting}

This single-center retrospective study was performed at the Second Xiangya Hospital of Central South University after approved by the ethics committee of the Second Xiangya Hospital of Central South University (ChiCTR.org Registration number: ChiCTR1900025821, 09/09/2019), all patients or their legal guardian provided informed consent for the usage of their clinical data and/or samples.

The full trial protocol can be accessed on the website of http://www.chictr.org.cn/index.aspx if available.

\section{Study population}

Children patients aged 1 to $\leq 18$ years who was administered voriconazole enterally or parenterally and monitored at least one voriconazole plasma trough concentration from between 1 st January 2016 and 31 st December 2018 was eligible for study. Patients with multiple hospitalization would be a cumulative statistic data.

Exclusion criteria were: out-patients, incomplete medical record information and body weight of $\geq 50 \mathrm{~kg}$ when patients aged of 12 to 14 years. 
From the electronic medical record information system, patients' demographic and clinical data were collected, including ethnicity, age, sex, body weight (BW), CYP2C19 genotype, underlying disease, treatment indication, site of infection, VRC dosing, route of administration, VRC trough concentrations, treatment duration, concomitant medications, adverse drug reactions, efficacy, liver function, and kidney function.

\section{VRC administration and TDM blood sampling}

Due to lacking of guidelines for the administration and monitoring of voriconazole in children, voriconazole dosing regimens were performed by clinicians considering recommending VRC package insert dose and the actual condition of patients in present study. Before the next dose, all TDM blood samples were collected at steady-state conditions. Repeat TDM was conducted by the clinician according to treatment response. The ideal target trough concentration of VRC was 1.0-5.5 $\mu \mathrm{g} / \mathrm{mL}^{9}$.

\section{Analysis of VRC trough concentration}

VRC plasma concentration was measured by an automatic two-dimensional liquid chromatography method reported in our previous paper ${ }^{10}$. The linearity range was 0.35 to $11.26 \mu \mathrm{g} / \mathrm{mL}$. The intra-day and inter-day precisions were $1.94-2.22 \%$ and $2.15-6.78 \%$, respectively.

\section{CPY2C19genotypes and phenotype assignment}

DNA was separated from the suspending white cells and was purified with the E.Z.N.A® SQ Blood DNA Kit II method. CYP2C19 genotypes was implemented by Sanger dideoxy DNA sequencing method with ABI3730xl-full automatic sequencing instrument ( $A B I$ Co., Carlsbad, California) from Boshang Biotechnology Co. Ltd. in Shanghai, China. According to definition of the Clinical Pharmacogenetics Implementation Consortium (CPIC) ${ }^{11}$, CYP2C19 phenotypes were categorized as several types based on $\operatorname{CYP} 2 C 19 * 1, * 2 * 3$, or $* 17$ allele nomenclature, including ultra-rapid metabolizer (UM), rapid metabolizer (RM), extensive metabolizer (EM), intermediate metabolizer (IM) and poor metabolizer (PM). Extensive metabolizers were considered as a normal or wild-type metabolizer of CYP2C19.

\section{Outcome and safety assessment}

The definition of IFI treatment was in accordance with the European Organization for Research and Treatment of Cancer/Invasive Fungal Infections Cooperative Group and the National Institute of Allergy and Infectious Diseases Mycoses Study Group (EORTC/MSG) ${ }^{12}$. Clinical outcomes of target antifungal therapy were categorized as "clinical successful" (complete or partial remission of clinically significant signs and symptoms and improvement of imaging manifestations at the end of treatment) or "clinical failure" (no response, disease progression or death due to IFI). Prophylaxis and empirical antifungal therapy were classified as a 'successful' without breakthrough fungal infection after discontinuing voriconazole treatment.

Based on the Common Terminology Criteria for Adverse Events (CTCAE) defined by the National Cancer Institute ${ }^{13}$, voriconazole-attributable adverse reaction such as visual disturbances, rash, vomiting, 
headache, elevated biomarkers of liver function, tachycardia and hallucinations at al. were categorized as a possible or stronger relationship event with voriconazole. Nevertheless, among them, increased liver biomarkers level, rash, and visual disturbances frequently resulted in voriconazole discontinuation.

\section{Statistical analysis}

Data was analyzed by SPSS (version 22.0; IBM Corp, Armonk, NY, USA). Continuous variables were expressed as median (range: quartiles). Categorical data was reported as frequencies and percentages. Using a Chi-squared test or Fisher's exact test and nonparametric Mann-Whitney $U$ test or Kruskal-Wallis $\mathrm{H}$ test, categorical data and continuous variables were compared, respectively. A P value (2-side) of $₫ 0.05$ was considered significant.

\section{Results}

\section{Demographics and clinical characteristics}

Ninety-four patients with a total of 145 VRC trough concentrations were enrolled in this retrospective study. Forty-nine of them were males. The median age was 7.5 years old. The primary diagnosis was hematological malignancy, accounting for $76.6 \%$, followed by respiratory infection $(69.1 \%)$ and bloodstream infection (38.3\%). The major infection sites were lung (66.0\%) and blood (25.5\%). Among the enrolled patients, only $15(19 \%)$ patients were diagnosed with confirmed IFI. Most of patients $(n=48)$ were administrated prophylactically, together with therapeutically $(20.2 \%)$ and empirically $(28.7 \%)$ respectively post-diagnosis. Totally, $80.8 \%$ of the patients achieved clinical success. Patients' demographic data and clinical characteristics are summarized in Table 1. 
Table 1

Patients' demographic data and clinical characteristics.

\begin{tabular}{|c|c|c|c|c|c|}
\hline \multirow[t]{2}{*}{ Characteristics } & \multirow{2}{*}{$\begin{array}{l}\text { Median } \\
\text { (quartile range or } \\
\text { percentage) }\end{array}$} & \multicolumn{4}{|c|}{ Age groups } \\
\hline & & $\leq 2 y$ & $2 y-6 y$ & $6 y-12 y$ & $12 y-18 y$ \\
\hline Age(y) & $7.5(4.0-14.2)$ & & & & \\
\hline Sex(male/female) & $49 / 45$ & $8 / 7$ & $12 / 16$ & $13 / 8$ & $16 / 14$ \\
\hline $\mathrm{BW}(\mathrm{kg})$ & $24.2(14.5-44.1)$ & $\begin{array}{l}11.5(9.2- \\
14.0)\end{array}$ & $\begin{array}{l}16.0(14.5- \\
18.0)\end{array}$ & $\begin{array}{l}30(23.5- \\
35.8)\end{array}$ & $\begin{array}{l}54.7(43.6- \\
61.8)\end{array}$ \\
\hline $\begin{array}{l}\text { Duration } \\
\text { hospitalization(d) }\end{array}$ & $31(23-46)$ & $\begin{array}{l}30(18- \\
43)\end{array}$ & $35(27-57)$ & $\begin{array}{l}34(24- \\
43)\end{array}$ & $25(20-42)$ \\
\hline \multicolumn{6}{|l|}{ Underlying conditions } \\
\hline $\begin{array}{l}\text { Hematological } \\
\text { malignancies }\end{array}$ & $72(76.6 \%)$ & 12 & 26 & 15 & 19 \\
\hline $\begin{array}{l}\text { Non-hematological } \\
\text { malignancies }\end{array}$ & $8(8.5 \%)$ & 2 & 2 & 3 & 1 \\
\hline Respiratory infections & $65(69.1 \%)$ & 8 & 23 & 15 & 19 \\
\hline $\begin{array}{l}\text { Bloodstream } \\
\text { infections }\end{array}$ & $36(38.3 \%)$ & 5 & 14 & 12 & 5 \\
\hline Fungal infection & $18(19.1 \%)$ & 3 & 5 & 8 & 2 \\
\hline $\begin{array}{l}\text { Allograft renal } \\
\text { transplantation }\end{array}$ & $9(9.6 \%)$ & 0 & 0 & 2 & 7 \\
\hline \multicolumn{6}{|l|}{ Infective sites } \\
\hline Lung & $62(66.0 \%)$ & 14 & 14 & 12 & 22 \\
\hline Blood & $24(25.5 \%)$ & 1 & 11 & 7 & 5 \\
\hline Oral mucosa & $1(1.0 \%)$ & 0 & 1 & 0 & 0 \\
\hline Perineum & $1(1.0 \%)$ & 0 & 1 & 0 & 0 \\
\hline Undefined & $6(6.4 \%)$ & 0 & 1 & 2 & 3 \\
\hline \multicolumn{6}{|l|}{ IFI diagnosis } \\
\hline Proven & $15(16.0 \%)$ & 2 & 5 & 2 & 6 \\
\hline Probable & $31(33.0 \%)$ & 8 & 8 & 9 & 6 \\
\hline Possible & $48(51.1 \%)$ & 5 & 15 & 10 & 18 \\
\hline
\end{tabular}

PPIs: proton pump inhibitors 


\begin{tabular}{|c|c|c|c|c|c|}
\hline \multirow[t]{2}{*}{ Characteristics } & \multirow{2}{*}{$\begin{array}{l}\text { Median } \\
\text { (quartile range or } \\
\text { percentage) }\end{array}$} & \multicolumn{4}{|c|}{ Age groups } \\
\hline & & $\leq 2 y$ & $2 y-6 y$ & $6 y-12 y$ & $12 y-18 y$ \\
\hline \multicolumn{6}{|l|}{ Treatment indication } \\
\hline Therapeutic & $19(20.2 \%)$ & 3 & 6 & 2 & 8 \\
\hline Empirical & $27(28.7 \%)$ & 6 & 8 & 7 & 6 \\
\hline Prophylactic & $48(51.1 \%)$ & 6 & 14 & 12 & 16 \\
\hline \multicolumn{6}{|l|}{ Clinical outcome } \\
\hline Success & $76(80.8 \%)$ & 12 & 23 & 17 & 24 \\
\hline Failure & $18(19.2 \%)$ & 3 & 5 & 4 & 6 \\
\hline \multicolumn{6}{|l|}{ Concomitant therapy } \\
\hline PPIs & $41(43.6 \%)$ & 3 & 12 & 8 & 18 \\
\hline Glucocorticoids & $27(28.7 \%)$ & 5 & 5 & 6 & 11 \\
\hline Antifungal drugs & $14(14.9 \%)$ & 4 & 2 & 2 & 6 \\
\hline $\begin{array}{l}\text { Immunosuppressive } \\
\text { agents }\end{array}$ & $9(9.6 \%)$ & 0 & 3 & 0 & 6 \\
\hline PPIs: proton pump ir & & & & & \\
\hline
\end{tabular}

\section{Voriconazole administration and therapeutic trough concentration}

For all patients, $5.2 \mathrm{mg} / \mathrm{kg}$ of voriconazole twice daily was the median level. The median dose of 7.1, 6.3, 5.2 and $3.4 \mathrm{mg} / \mathrm{kg}$ twice daily was administered for subgroup by age of $\leq 2,2-6,6-12$, and $12-18$ years respectively. For them, children aged $12-18$ had the highest maintenance dose $(P<0.001)$ (Fig. 1). Except for children aged $\leq 2 y$, children had lower maintenance dose than the recommended dose levels. The medication duration and time of initial VRC TDM varied widely, median of 17.5 days (11-26.2 days) and 7 days (3.8-11 days), respectively. Patients' voriconazole administration showed in Table 2. 
Table 2

Patients' voriconazole administration and TDM data.

\begin{tabular}{|c|c|c|c|c|c|}
\hline \multirow[t]{2}{*}{ Characteristics } & \multirow{2}{*}{$\begin{array}{l}\text { Mean } \\
\text { (quartile range or } \\
\text { percentage) }\end{array}$} & \multicolumn{4}{|l|}{ Age groups } \\
\hline & & $\leq 2 y$ & $2 y-6 y$ & $6 y-12 y$ & $12 y-18 y$ \\
\hline $\begin{array}{l}\text { Duration VRC } \\
\text { using(d) }\end{array}$ & $17.5(11-26.2)$ & $16(11-29)$ & $\begin{array}{l}22(12.2- \\
33.7)\end{array}$ & $\begin{array}{l}21(15.5- \\
24)\end{array}$ & $13(8-24)$ \\
\hline \multicolumn{6}{|l|}{$\begin{array}{l}\text { Administration } \\
\text { route }\end{array}$} \\
\hline Oral & 83(88.3\%) & 12 & 27 & 20 & 24 \\
\hline Intravenous & $11(11.7 \%)$ & 3 & 1 & 1 & 6 \\
\hline $\begin{array}{l}\text { Initial sampling } \\
\text { time(d) }\end{array}$ & $7(3.8-11)$ & $7(3-10)$ & $8(5-13)$ & $8(5-19)$ & $4.5(3-6.2)$ \\
\hline $\begin{array}{l}\text { VRC initial } \\
C_{\text {trough }}(\mu \mathrm{g} / \mathrm{mL})\end{array}$ & $1.56(0.30-3.38)$ & $\begin{array}{l}0.17(0.11- \\
2.1)\end{array}$ & $\begin{array}{l}0.87(0.11- \\
2.86)\end{array}$ & $\begin{array}{l}2.45(0.69- \\
7.30)\end{array}$ & $\begin{array}{l}2.14(1.05- \\
3.19)\end{array}$ \\
\hline$<1.0$ & $38(40.5 \%)$ & 9 & 16 & 7 & 6 \\
\hline $1.0-5.5$ & $43(45.7 \%)$ & 6 & 10 & 6 & 21 \\
\hline$>5.5$ & $13(13.8 \%)$ & 0 & 2 & 8 & 3 \\
\hline $\begin{array}{l}\text { VRC } \\
C_{\text {trough }}(\mu \mathrm{g} / \mathrm{mL})\end{array}$ & $1.58(0.54-3.12)$ & $\begin{array}{l}0.18(0.11- \\
2.21)\end{array}$ & $\begin{array}{l}1.19(0.22- \\
3.27)\end{array}$ & $\begin{array}{l}2.02(0.86- \\
5.78)\end{array}$ & $\begin{array}{l}\text { 2.02.(1.02- } \\
3.12)\end{array}$ \\
\hline
\end{tabular}

In all blood samples, $54.5 \%$ of them achieved the target concentrations; however, $35.9 \%$ were subtherapeutic and $9.6 \%$ were super-therapeutic post-multiple dose. 62.8.\% (59 in 94) of patients achieved one or more therapeutic level. As showed in Fig. 2, the disparate and weight-adjusted VRC dose was prescribed in age groups owing to achieve therapeutic concentrations $(P<0.001)($ Fig. 2). However, we interestingly found that patients achieved sub-therapeutic concentrations $(n=9)$ had a higher maintenance doses compared with those achieved therapeutic level $(n=6)$ in group aged $\leq 2$ years $(7.1$ vs $5.8 \mathrm{mg} / \mathrm{kg}$ ).

Oral administration of voriconazole $(n=83)$ was the most common route in our study. But no significant difference was found between the maintenance dose and administration route $(P=0.852)$ (Fig. 2$)$. Besides that, co-administration was also an important factor affecting VRC trough concentration. In our research, the group of combining PPI required higher dose in achieving therapeutic level compared with non-PPI group $(P=0.001)$ (Fig. 3). The inter-individual variability of the initial trough concentration in 94 patients was $120.6 \%$ (94 initial trough concentrations), and the intra-individual variability was $61.1 \%$ 
(33.1\%-103.5\%) (21 patients with the same dose, the same administration route and TDM frequency $\geq 2$ times) (Fig. 4).

\section{CYP2C19genotyping and VRC exposure}

Forty-five patients obtained CYP2C19 genotype with a total 93 trough concentration, among which EM metabolizers (CYP2C19*1/*1), IM metabolizers $(C Y P 2 C 19 * 1 / * 2, C Y P 2 C 19 * 1 / * 3)$ and PM metabolizers $($ CYP2C19*2/*2, CYP2C19*2/3) counted of $15(33.3 \%), 25(55.6 \%)$ and $5(11.1 \%)$, respectively. There are obvious relationships between trough concentrations and CYP2C19 genotypes, with the VRC initial trough concentration of $1.69 \mu \mathrm{g} / \mathrm{mL}, 3.23 \mu \mathrm{g} / \mathrm{mL}$ and $4.15 \mu \mathrm{g} / \mathrm{mL}$ in EM, IM and PM metabolizers respectively. The initial trough concentrations were significantly decreased in EM metabolizers (CYP2C19*1/*1 carriers) compared with IM and PM metabolizers, but no statistical difference was found. Due to the small size of PM genotypes, we combined them with IM genotypes. The rate of reaching initial target trough concentration is shown in Figure.5. And the maintenance dose reaching target concentration range was significantly higher compared EM metabolizers with IM/PM metabolizers, but there was no statistical difference $(6.4 \mathrm{mg} / \mathrm{kg}$ vs $4.8 \mathrm{mg} / \mathrm{kg}, \mathrm{P}=0$.

\section{Dosing adjusting based on TDM}

According to the results of TDM, a total of 23 patients (27 samples) in 94 patients had dose regimen adjustments, among which 19 patients were adjusted once and 4 patients adjusted twice.

Ten samples significant increased dose of $3.2 \mathrm{mg} / \mathrm{kg} / \mathrm{d}$ based on the lower VRC trough concentration (below $1.0 \mu \mathrm{g} / \mathrm{mL}$ ). Five patients with mean trough concentration of $4.77 \mu \mathrm{g} / \mathrm{mL}(4.15-5.32 \mu \mathrm{g} / \mathrm{mL}$ ) also adjusted dose regimen, decreasing dose by $3.4 \mathrm{mg} / \mathrm{kg} / \mathrm{d}(2.0-4.2 \mathrm{mg} / \mathrm{kg} / \mathrm{d})$ in four but increasing dose in one. A large proportion of Patients with high trough concentration adjusted their medication, among which VRC was discontinued in 3 samples and VRC dose was reduced by $4.2 \mathrm{mg} / \mathrm{kg} / \mathrm{d}(3.0-8.6 \mathrm{mg} / \mathrm{kg} / \mathrm{d})$ in 8 samples.

\section{Outcome and Safety}

Outcome data in patients with therapeutic, empirical or prophylactic treatment were analyzed. There was no significant difference in VRC dosing when compared clinical successful and clinical failure categorized by treatment indication (empirical/therapeutic: $P=0.867$, prophylactic: $P=0.762$ ) (Fig. 6$)$. In patients with empirical/therapeutic course, VRC maintenance dose of $5.0 \mathrm{mg} / \mathrm{kg}$ required for successful outcome $(34 / 46 ; 73.9 \%)$. For prophylactic treatment, 42 patients achieved successful outcome at median dose of $5.2 \mathrm{mg} / \mathrm{kg}(4.0-6.4 \mathrm{mg} / \mathrm{kg})$. No significant difference was found between empirical/therapeutic group and prophylactic group.

Three of 94 patients occurred voriconazole-related adverse reactions, 2 of them with persistent elevation of alanine aminotransferase (ALT) and/or aspartate aminotransferase (AST) and vomiting just in one. One patient with trough concentration of $5.32 \mu \mathrm{g} / \mathrm{mL}$ gradually recovered to normal liver function after drug withdrawal and the other one also showed normal liver function by reducing VRC dosage. For one 
patient administered voriconazole tablets, the symptoms of vomiting disappeared after intravenous administration. All the 3 patients finally achieved clinical effectiveness.

\section{Discussion}

Hematological malignant tumor patients had a high-risk of fungal infection due to immunodeficiency and chemotherapy drugs. Voriconazole is increasingly used for the prevention and treatment of IFI. The population pharmacokinetic characteristics of voriconazole in children were more complicated ${ }^{14}$ and showed a large individual variation. Therefore, this retrospective study highlighted the reasons and factors affected VRC therapeutic trough concentration in children and discussed the dose adjustment strategy of voriconazole.

At the current maintenance dose for children younger than 12 years, voriconazole shows nonlinear pharmacokinetics ${ }^{15,16}$, warranting more cautious dose adjustment. Our study showed a large variability in voriconazole trough levels, with rate of $120.6 \%$ and $61.1 \%$ in inter-individual and intra-individual variability, similar to the published study ${ }^{17}$. More than $50 \%$ of children could not reach the target range $(1.0-5.5 \mu \mathrm{g} / \mathrm{mL})$ at the initial trough concentration, which was consistent with many previous studies 9,18 , but was different with a European research ${ }^{6}$. The initial trough concentration in children aged 2-12 was significantly higher than that in children aged under 2 years, suggested a faster metabolism for infants. The EMA approved higher doses in 2 to 12 years old children, an $8 \mathrm{mg} / \mathrm{kg}$ intravenously twice daily (9 $\mathrm{mg} / \mathrm{kg}$ day 1 ) or a $9 \mathrm{mg} / \mathrm{kg}$ orally twice daily. Our data suggested that underdose was so prevalent that $40.5 \%$ of children failed to reach the target concentration range and voriconazole dose was inadequate in this population. Lacking guidelines for dose recommendation of voriconazole in children, voriconazole maintenance doses vary widely in our center. As compared to the research of Karlsson $\mathrm{MO}$ et $\mathrm{al}^{7}$ and Neely $\mathrm{M}$ et al ${ }^{19}$ who illustrated VRC optimal dose of $7 \mathrm{mg} / \mathrm{kg}$ twice daily in children 2 to 12 years old, Shima $\mathrm{H}$ et al ${ }^{20}$ who recommended at least $8.5 \mathrm{mg} / \mathrm{kg}$ twice daily of VRC optimal dose for patients younger than 2 years, children with leukemia in our center might require higher doses and more frequent monitoring. The primary administration route of voriconazole in the present study was oral administration (88.3\%) rather than the intravenous route recommended by the package insert for VRC. However, variability in oral bioavailability caused by meals and hepatic first-pass effect might associate with lower drug exposure ${ }^{3,21}$, which could be the reason for sub-therapeutic trough concentration.

Similar to that reported (7-10 days) in other centers ${ }^{19}$, the average time of the first TDM blood sampling in our hospital was 7 days (3.8-11days). In a randomized controlled trial ${ }^{22}$, the first TDM blood sampling was done on the fourth day after the initiation of voriconazole, which was calculated based on data from the literature so that it coincided with the target trough concentration range (1.0-5.5 mg/L). However, Miyakis S et.al ${ }^{23}$ showed that the initial trough concentrations of $\leq 0.35 \mu \mathrm{g} / \mathrm{mL}$ were significantly associated with increased mortality in pediatric with invasive candidiasis, so it was advised that the first steady-state TDM should be done ideally as early as possible (day 3 of therapy) to allow prompt dose adjustment. Our findings are consistent with previous study ${ }^{24}$, the co-administration of PPTs, mainly 
omeprazole, could significantly increase voriconazole plasma concentrations through CYP2C19 inhibition. However, in children the further exploration of the clinical implication of this drug interaction is absolutely imperative.

Another important factor affecting VRC therapeutic trough concentrations was polymorphism of CYP2C19. The CYP2C19 genotypic and phenotypic variability were extensive among different ethnic groups, but was controversial in relation to voriconazole treatment. Generally, the Caucasians or Africans have a lower proportion of PM metabolizers than Asians (2-5\%, $6 \%$ and $13-23 \%$, respectively). And for Caucasians and Africans, it is about 4 times more proportion in the Asian than them among the CYP2C19*17 allele ${ }^{25-29}$. No CYP2C19*17 allele was found in our study. And EMs patients had a higher VRC trough concentrations and rate of VRC target concentrations. The above situation indicated that CYP2C19 genotype had a great influence on voriconazole metabolism in children, and the dose should be increased in EM patients. Hicks $\mathrm{JK}$ et al ${ }^{30}$ emphasized that a starting dose above $14 \mathrm{mg} / \mathrm{kg} /$ day could be recommended for all pediatric patients except for CYP2C19 extensive metabolizers. Based on age and CYP2C19 genotypes, VRC dose regime could be better determined and variation of drug trough concentrations could dramatically decrease. Therefore, available CYP2C19 genotype before the initial administration of voriconazole could improve the accuracy and safety of initial dosing ${ }^{31}$.

A guideline for VRC dose optimization is imperative in pediatric patients. A randomized controlled trial implemented by Park WB et al ${ }^{22}$ developed a dose adjustment strategy as following: increasing another $100 \%$ doses when the trough level was $<1.0 \mathrm{mg} / \mathrm{L}$, on the contrary, reducing doses by $50 \%$ when the trough level was $>5.5 \mathrm{mg} / \mathrm{L}$, but if there were adverse events, withholding voriconazole and reduce subsequent doses. However only $45.1 \%$ patients in 51 patients whose concentrations were not reach the target had the dose adjustment. And almost all the children younger than 2 failed to reach target concentration in the first TDM. Whether this optimized dosage strategy applicable to children $\leq 2$ years old was unclear. Zembles TN et al ${ }^{32}$ had described their VRC optimized dosing strategy in three patients younger than 2 years and emphasized that a higher initial dose and perhaps 8 hours interval dosing schedule should be given to achieve VRC target therapeutic concentrations. Besides, Kendre'a $\mathrm{M}$ et al ${ }^{33}$ showed a regimen of $6 \mathrm{mg} / \mathrm{kg}$, 3 times per day for intravenous and had proved its safety in a preterm infant younger than 3 months. Because voriconazole is not routinely monitored in children in our hospital, VRC trough concentration was seldom monitored again after adjusting dose. Finally, the dose-adjusted treatment response was significantly improved compared with the initial response. Pediatrician should recognize the importance of therapeutic drug monitoring of VRC and refer to the interpretation results of TDM conducted by clinical pharmacists in dosage adjustment.

Voriconazole, given orally or intravenously, is well tolerated in most of patients, with a rate of adverse reactions of $3.2 \%$, which is much lower than previous reported $(20.0 \%)^{18}$. Visual disturbances and photosensitive skin reactions were difficult to be detected and explained in pediatric may be one reason for that lower rate of adverse reactions. 
Our study is a single-center retrospective study. There are a few limitations in study design and analysis. First, too small sample size restricts the difference significance analysis.

Second, no uniform guidelines and standardized protocol for VRC dose adjustment based on TDM data, resulting in a few confusions in doctors when adjusting doses.

Third, the number of CYP2C19 genotypic and phenotypic is too small to detect difference between trough concentration and genotypes.

\section{Conclusion}

In conclusion, there are large inter-individual and intra-individual variability with voriconazole trough concentration in children, and many factors could affect VRC therapeutic trough concentration, including age, dose, time of TDM sampling, PPI coadministration and CYP2C19 genotype. Younger pediatric patients might need to have a higher dosage regime. The early, routine and repeat TDM of trough concentration is extremely necessary in order to ensure safety and effective treatment. It is crucial to determine the initial dose of voriconazole based on CYP2C19 genotype data. Therefore, a large size, multi-center prospective studies is imperative to identify and validate dose-optimization strategies for pediatric patients in the future.

\section{Declarations}

\section{Acknowledgements}

The authors would like to acknowledge Xiao-Bin Lin, Zi-Wei Li, Zhu-Feng Wu, Jia-Min Wu, Xiang-Xin Wu for assistance in DNA extraction, collection of clinical data and blood samples. We also would like to thank Doctor Hua-Lin Cai and Doctor Mou-Ze Liu for helping with the concentration analysis.

\section{Author Contributions}

Yi-chang Zhao and Yang Zou wrote the main manuscript.

Bi-kui Zhang, Miao Yan,and Yi-chang Zhao designed research

Dan Tang, Yi-wen Xiao, Feng Wang, Da-xiong Xiang performed the statastic analysis.

Feng Yu and Chen-lin Xiao reviewed the manuscript.

\section{Data Availability}

The datasets generated during and/or analyzed during the current study are available by request.

\section{Funder}


This study was supported by Hunan Pharmaceutical Association of China under Grant [HMA202001002]. The authors have indicated that they have no conflicts of interest regarding the content of this article.

\section{Statement}

The research was approved by the ethics Committee of the Second Xiangya Hospital of Central South University and was registered in the website of http://www.chictr.org.cn/index.aspx with ChiCTR.org Registration number of ChiCTR1900025821 (09/09/2019). I declare that all methods were carried out in accordance with relevant guidelines and regulations in the manuscript on behalf of all the other authors.

\section{References}

1. Lehrnbecher, T. et al. Guideline for the management of fever and neutropenia in children with cancer and hematopoietic stem-cell transplantation recipients: 2017 Update. J Clin Oncol. 35, 20822094(2017).

2. Fisher, B. T. et al. Risk factors for invasive fungal disease in pediatric cancer and hematopoietic stem cell transplantation: A systematic review. J Pediatric Infect Dis Soc. 7, 191-198(2018).

3. Denning, D. W. et al. Efficacy and safety of voriconazole in the treatment of acute invasive aspergillosis. Clin Infect Dis. 34, 563-571(2002).

4. Hoban, D. J., Zhanel, G. G. \& Karlowsky, J. A. In vitro susceptibilities of Candida and Cryptococcus neoformans isolates from blood cultures of neutropenic patients. Antimicrob Agents Chemother. 43. 1463-1464 (1999).

5. Tissot, F. et al. ECIL-6 guidelines for the treatment of invasive candidiasis, aspergillosis and mucormycosis in leukemia and hematopoietic stem cell transplant patients. Haematologica. 102, 433-444(2017).

6. Pascual, A. et al. Voriconazole therapeutic drug monitoring in patients with invasive mycoses improves efficacy and safety outcomes. Clin Infect Dis. 46, 201-211(2008).

7. Karlsson, M. O., Lutsar, I. \& Milligan, P. A. Population pharmacokinetic analysis of voriconazole plasma concentration data from pediatric studies. Antimicrob Agents Chemother. 53, 935944(2009).

8. Groll, A. H. et al. Fourth european conference on infections in leukaemia (ECIL-4): guidelines for diagnosis, prevention, and treatment of invasive fungal diseases in paediatric patients with cancer or allogeneic haemopoietic stem-cell transplantation. Lancet Oncology. 15, e327-340(2014).

9. Liu, L. et al. Dose optimisation of voriconazole with therapeutic drug monitoring in children: a singlecentre experience in China. Int J Antimicrob Agents. 49, 483-487(2017).

10. Tang, D. et al. Identifying factors affecting the pharmacokinetics of voriconazole in patients with liver dysfunction: A population pharmacokinetic approach. Basic Clin Pharmacol Toxicol.125, 3443(2019). 
11. Moriyama, B. et al. Clinical pharmacogenetics implementation consortium (CPIC) guidelines for CYP2C19 and voriconazole therapy. Clin Pharmacol Ther. 102, 45-51(2017).

12. De, P. B. et al. Revised definitions of invasive fungal disease from the European Organization for Research and Treatment of Cancer/Invasive Fungal Infections Cooperative Group and the National Institute of Allergy and Infectious Diseases Mycoses Study Group (EORTC/MSG) consensus group. Clin Infect Dis. 46, 1813-1821(2008).

13. National Cancer Institute. Common Terminology Criteria for Adverse Events (CTCAE) v 5.0. https://ctep.cancer.gov/protocolDevelopment/electronic_applications/ctc.htm/ (2018)

14. Neely, M. et al. Achieving target voriconazole concentrations more accurately in children and adolescents. Antimicrob Agents Chemother. 59, 3090-3097(2015).

15. Walsh, T. J. et al. Pharmacokinetics, safety, and tolerability of voriconazole in immunocompromised children. Antimicrob Agents Chemother. 54. 4116-4123 (2010).

16. Michael, C. et al. Voriconazole pharmacokinetics and safety in immunocompromised children compared to adult patients. Antimicrob Agents Chemother. 54, 3225-3232(2010).

17. Hu, L. et al. Therapeutic drug monitoring of voriconazole in children from a tertiary care center in China. Antimicrob Agents Chemother. 62, 955-963(2018).

18. Boast, A., Curtis, N., Cranswick, N. \& Gwee, A. Voriconazole dosing and therapeutic drug monitoring in children: experience from a paediatric tertiary care centre. J Antimicrob Chemother.71, 20312036(2016).

19. Neely, M., Rushing, T., Kovacs, A., Jelliffe, R. \& Hoffman, J. Voriconazole pharmacokinetics and pharmacodynamics in children. Clin Infect Dis. 50, 27-36(2010).

20. Shima, H., Miharu, M., Osumi, T., Takahashi, T. \& Shimada, H. Differences in voriconazole trough plasma concentrations per oral dosages between children younger and older than 3 years of age. Pediatr Blood Cancer. 54, 1050-1052(2010).

21. Boyd, A. E. et al. Adverse reactions to voriconazole. Clin Infect Dis. 39, 1241-1244(2004).

22. Park, W. B. et al. The effect of therapeutic drug monitoring on safety and efficacy of voriconazole in invasive fungal infections: a randomized controlled trial. Clin Infect Dis. 55, 1080-1087(2012).

23. Miyakis, S., van Hal, S. J., Ray, J. \& Marriott, D. Voriconazole concentrations and outcome of invasive fungal infections. Clin Microbiol Infect. 16, 927-933(2010).

24. Boyd, N. K., Zoellner, C. L., Swancutt, M. A. \& Bhavan, K. P. Utilization of omeprazole to augment subtherapeutic voriconazole concentrations for treatment of Aspergillus infections. Antimicrob Agents Chemother.56, 6001-2(2012).

25. Chen, L. et al. Genetic polymorphism analysis of CYP2C19 in Chinese Han populations from different geographic areas of mainland China. Pharmacogenomics. 9, 691-702(2008).

26. Li-Wan-Po, A., Girard, T., Farndon, P., Cooley, C. \& Lithgow, J. Pharmacogenetics of CYP2C19: functional and clinical implications of a new variant CYP2C19*17. Br J Clin Pharmacol. 69, 222230(2010). 
27. Mikus, G., Scholz, I. M. \& Weiss, J. Pharmacogenomics of the triazole antifungal agent voriconazole. Pharmacogenomics. 12, 861-872(2011).

28. Strom, C. M. et al. Testing for variants in CYP2C19: population frequencies and testing experience in a clinical laboratory. Genet Med.14, 95-100(2012).

29. Goldstein, J. A. Clinical relevance of genetic polymorphisms in the human CYP2C subfamily. $\mathrm{Br} J$ Clin Pharmacol. 52. 349-355 (2001).

30. Hicks, J. K. et al. Voriconazole plasma concentrations in immunocompromised pediatric patients vary by CYP2C19 diplotypes. Pharmacogenomics. 15, 1065-1078(2014).

31. Friberg, L. E., Ravva, P., Karlsson, M. O. \& Liu, P. Integrated population pharmacokinetic analysis of voriconazole in children, adolescents, and adults. Antimicrob Agents Chemother.56, 30323042(2012).

32. Zembles, T. N., Thompson, N. E., Havens, P. L., Kaufman, B. A. \& Huppler, A. R. An optimized voriconazole dosing strategy to achieve therapeutic serum concentrations in children younger than 2 years old. Pharmacotherapy. 36. 1102-1108 (2016).

33. Muldrew, K. M., Maples, H. D., Stowe, C. D. \& Jacobs, R. F. Intravenous voriconazole therapy in a preterm infant. Pharmacotherapy. 25, 893-898(2005).

\section{Figures}

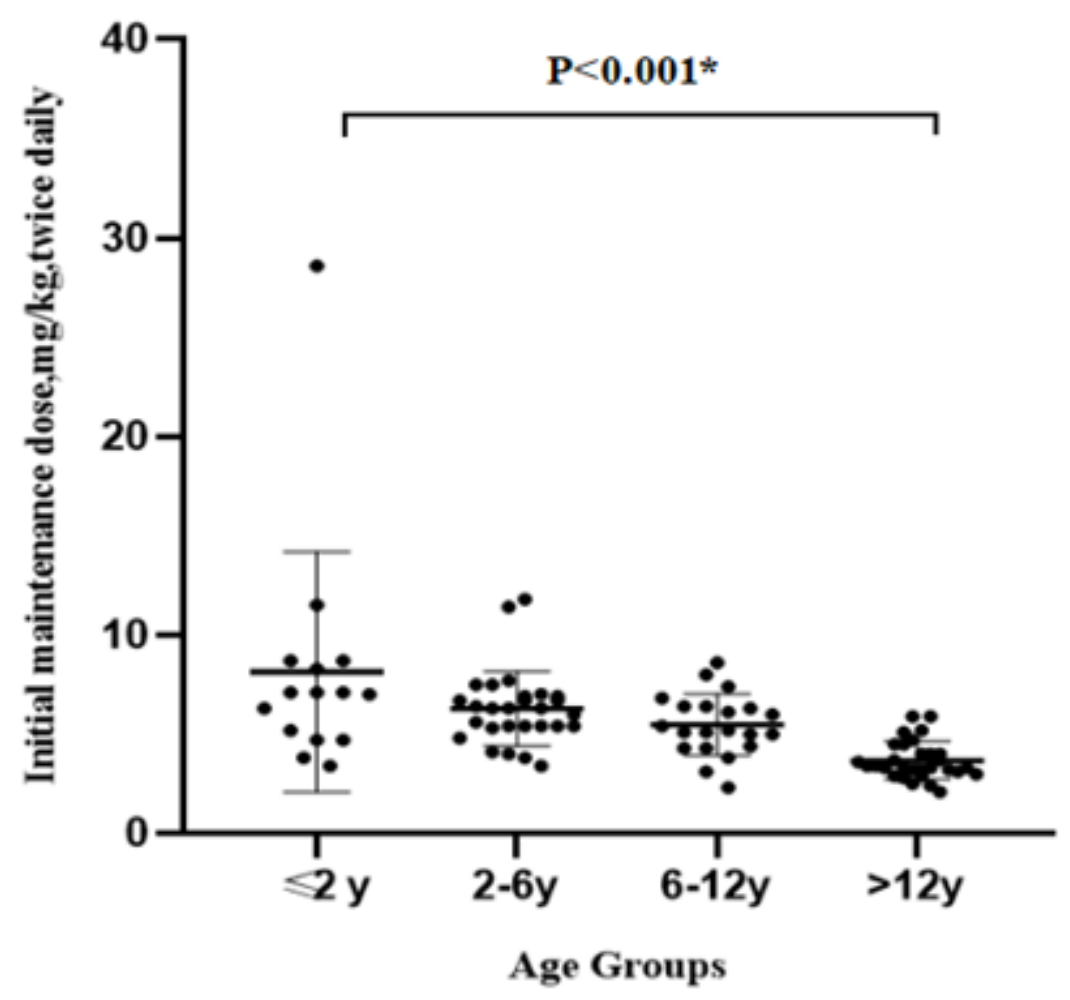

Figure 1 
Distribution of voriconazole initial maintenance dose at different age groups $(n=94)$, each point represents the dose for each patient.

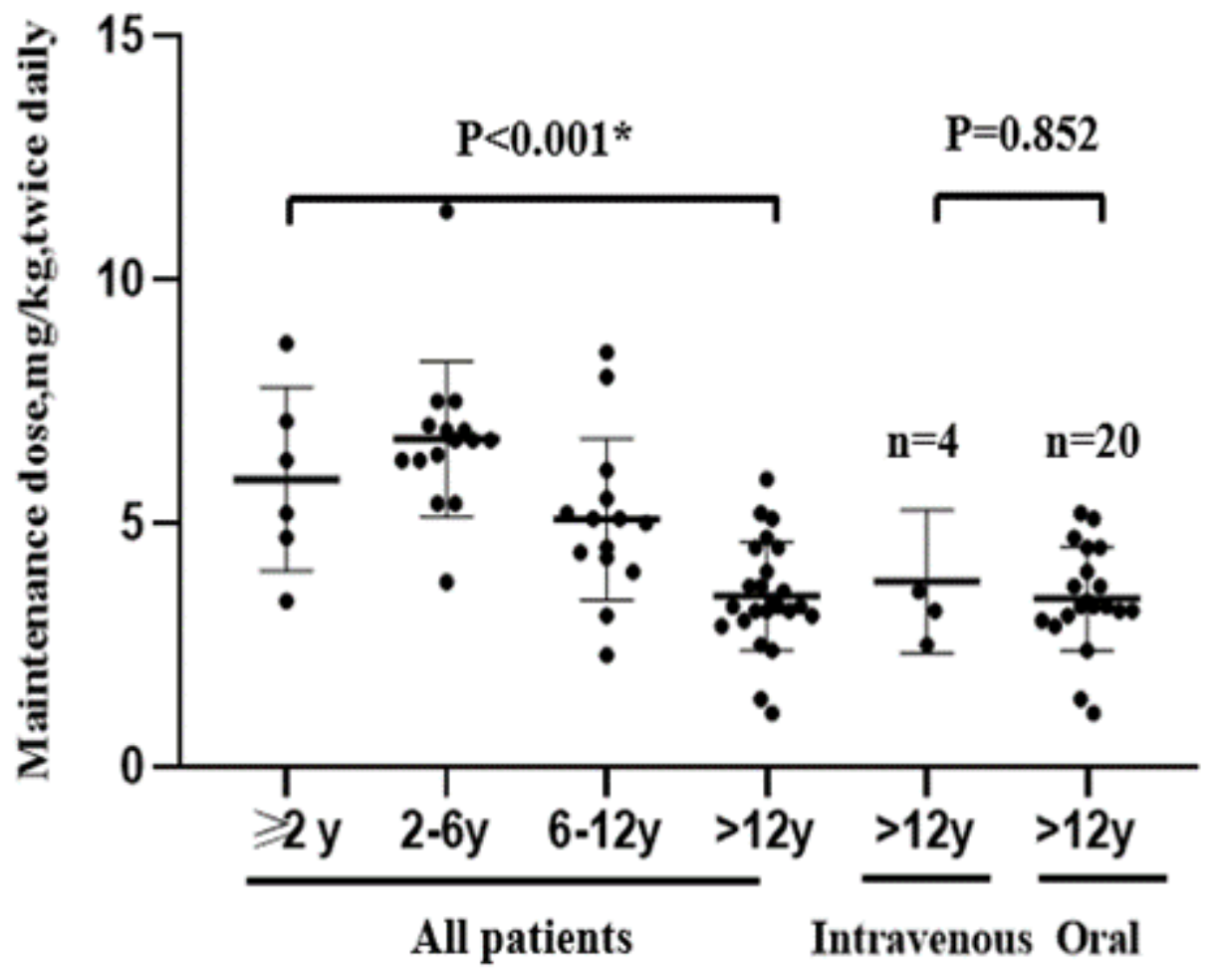

Figure 2

VRC maintenance doses required to achieve at least one therapeutic trough concentration $(1.0-5.5 \mu \mathrm{g} / \mathrm{mL})$ categorized by age and route of administration $(n=59)$.

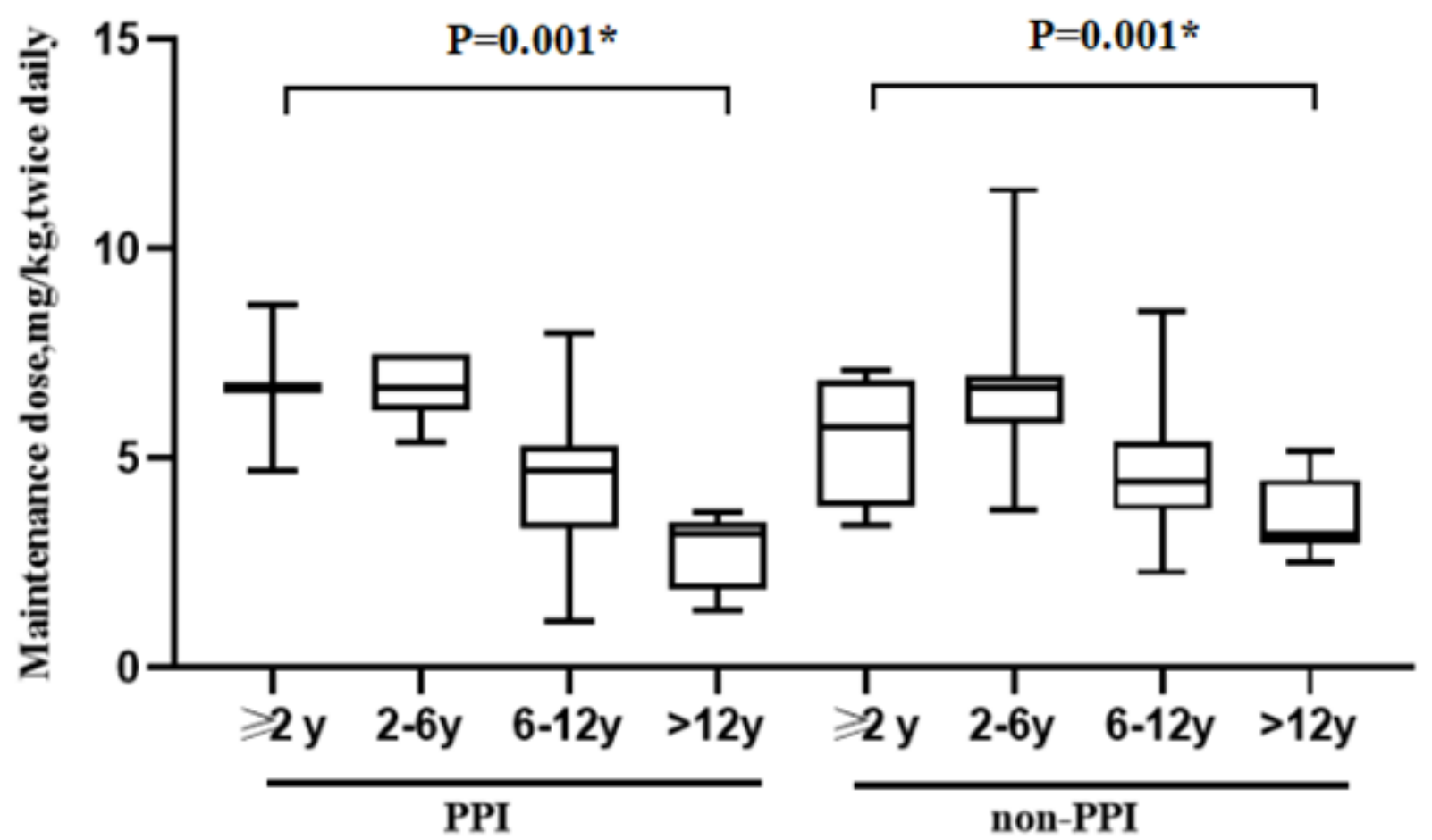

Figure 3 
Box plot of VRC maintenance dose requiring to achieve at least one therapeutic trough concentration (1.0$5.5 \mu \mathrm{g} / \mathrm{mL}$ ) categorized by age and PPI coadministration, which was represented by median, minimum, maximum, and interquartile range.

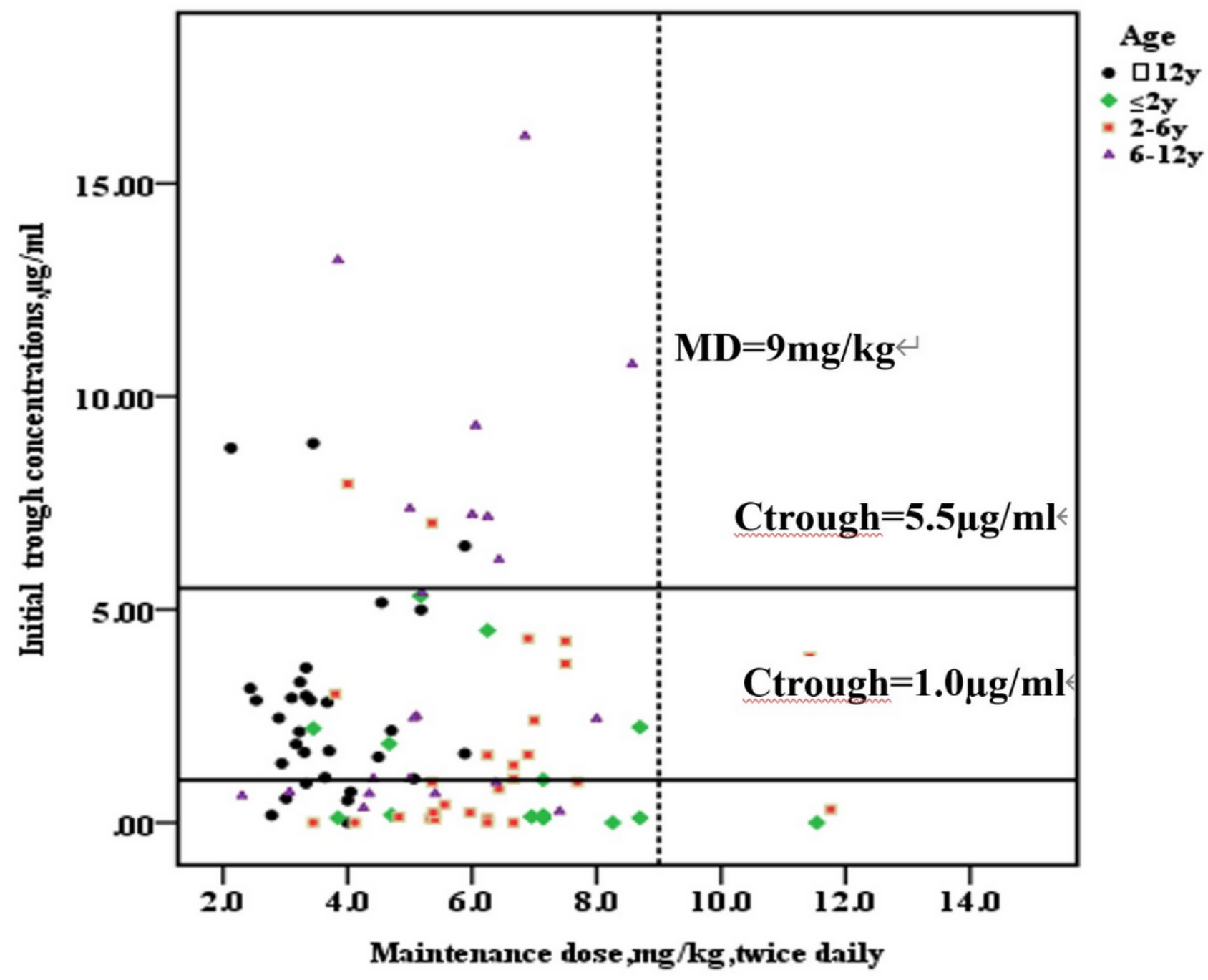

Figure 4

Distribution and interpatient variability of initial voriconazole trough concentrations at different weightadjusted maintenance doses $(n=94)$. 


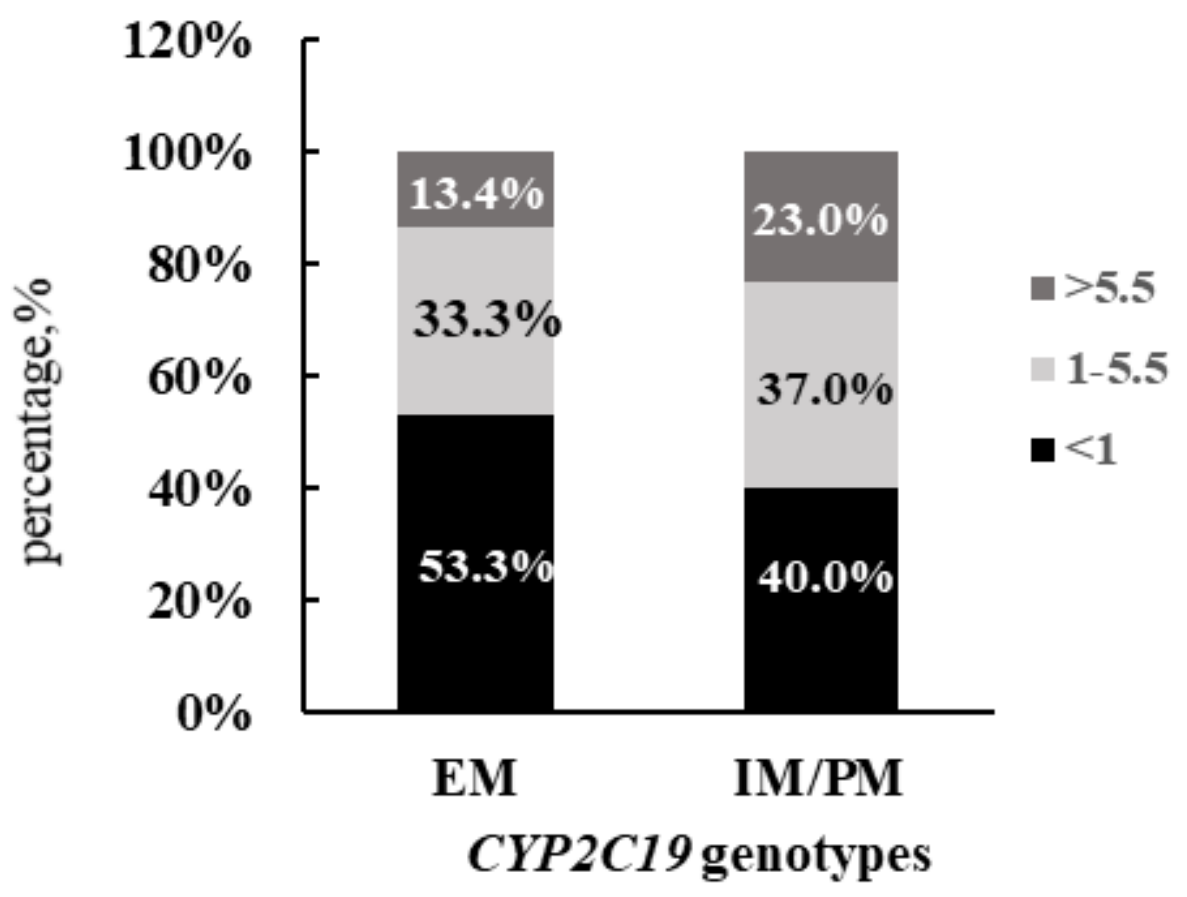

Figure 5

Proportion of three CYP2C19 genotypes with sub-therapeutic, therapeutic, and super-therapeutic voriconazole initial Ctrough $(n=45)$.

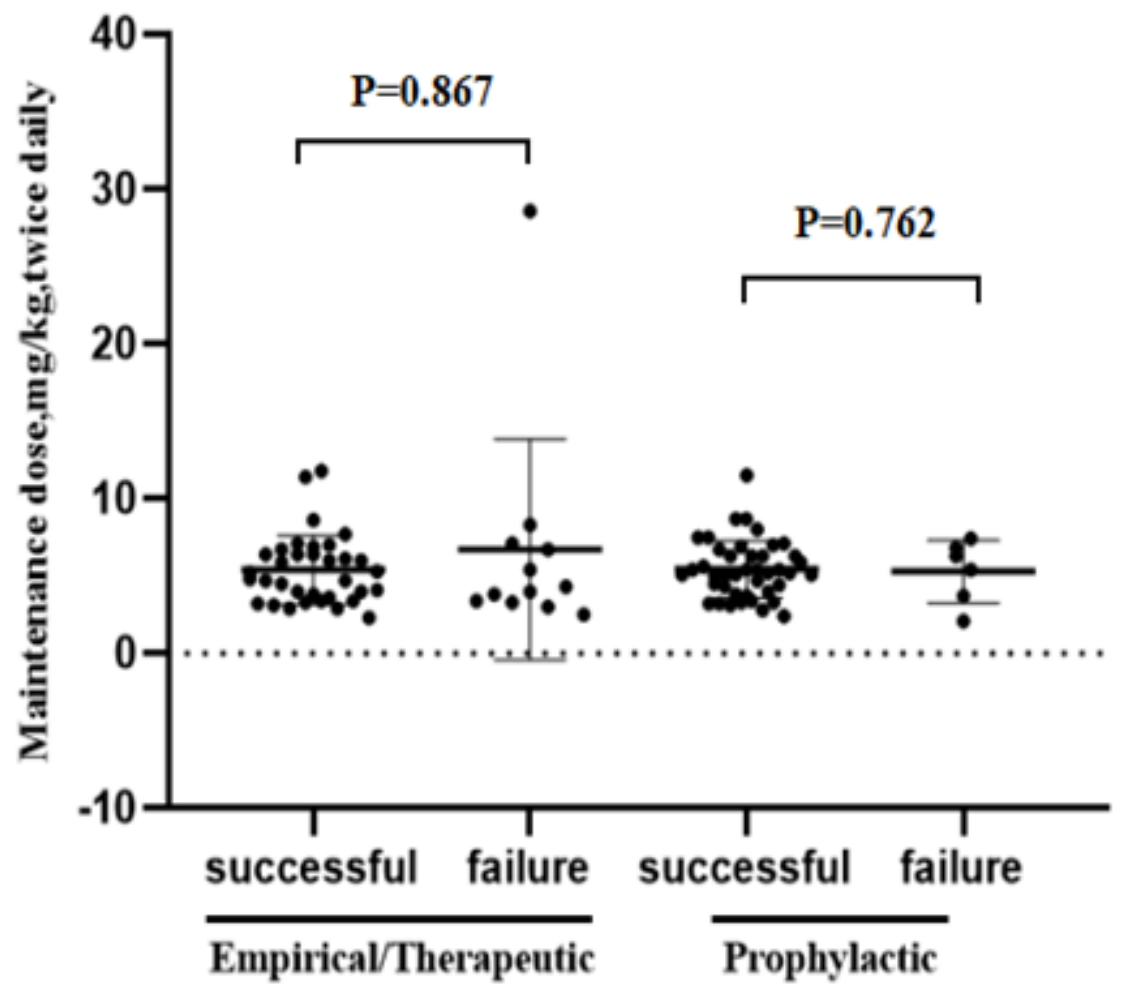

Figure 6 
VRC maintenance doses required to achieve clinical successful and clinical failure categorized by treatment indication. 